\title{
FACTORES URODINÁMICOS ASOCIADOS AL REFLUJO VESICOURETERAL EN EL ADULTO.
}

\author{
Miguel Vírseda Chamorro', 2, Ramón Diz Rodríguez', Jesús Salinas Casado², Ignacio Arance \\ Gill y Pedro Paños Lozano'.
}

Servicio de Urología'. Hospital Central de la Defensa. Madrid.

Unidad de Urodinámica². Hospital Ruber Internacional. Madrid. España.

Resumen.- OBJETIVO: La disfunción del tracto urinario inferior puede repercutir sobre el tracto urinario superior con la producción de reflujo vesicoureteral. El objetivo de nuestro estudio es valorar qué tipo de alteraciones del tracto urinario inferior se asocian a reflujo vesicoureteral en el adulto.

MÉTODOS. Se realizó un estudio transversal l"cross sectional study") en una muestra de 236 pacientes ( 149 varones y 87 mujeres), de edad media 45, 5 años (desviación típica 19,0 años), remitidos para estudio videourodinámico por síntomas funcionales del tracto urinario inferior. Los pacientes fueron sometidos a una historia clínica, exploración neurourológica, cistomanometría asociada a videocistografía de llenado, estudio
Miguel Vírseda Chamorro C/Argentina 4 - 1ㅇ - 1ำ 28027 Madrid. (España) bgmeli@terra.es Trabajo recibido: 4 de marzo 2006 presión/ flujo asociado a videocistografía miccional y electromiografía selectiva del esfínter periuretral. El análisis estadístico se realizó mediante el test de la chicuadrado.

RESULTADOS: Se observó la presencia de reflujo vesicoureteral en 19 pacientes $(8,1$ \% de la muestra). En 15 casos (79\%) el reflujo tuvo lugar durante el llenado, en 3 casos (16\%) durante la micción, y en 1 caso (5\%) en ambas fases. Se comprobó la existencia de una disfunción neurógena vesicouretral en 8 casos (42 \%), no existió disfunción neurógena vesicouretral en 11 casos (58\%). El reflujo vesicoureteral se clasificó como pasivo primario (sin alteración urodinámica que lo justifique) en 1 caso (5\%), pasivo con acomodación disminuida en 4 casos $(21 \%)$, pasivo evolutivo en 3 casos $(16 \%)$, activo involuntario en 7 casos (38\%), activo asociado a obstrucción orgánica del TUI en 1 caso (5\%), activo asociado a potencia contráctil elevada en 1 caso (5\%), y por prensa abdominal en 2 casos (10\%). Se comprobó que las únicas alteraciones urodinámicas asociadas a un aumento significativo de la prevalencia de reflujo vesicoureteral fueron: una presión de llenado, a máxima capacidad, superior a 12,5 $\mathrm{cm} \mathrm{H}_{2} \mathrm{O}$ lque aumentó la prevalencia de reflujo 7 veces) y la micción con prensa abdominal que aumentó la prevalencia de reflujo 2,8 veces.

CONCLUSIONES: La mayoría de los pacientes con sintomas funcionales del tracto urinario inferior y reflujo vesicoureteral asociado, presentan disfunciones del tracto urinario inferior que podrían justificar su reflujo. Sin embargo, sólo se comprobó un aumento estadísticamente significativo de la prevalencia de reflujo vesicoureteral en los pacientes con presión de llenado a máxima capacidad superior a $12,5 \mathrm{~cm} \mathrm{H}_{2} \mathrm{O}$ y en aquellos que realizaban la micción con prensa abdominal. 
Palabras clave: Reflujo vesicoureteral. Urodinámica. Factores de riesgo.

Summary.- OBJECTIVES: Lower urinary tract dysfunction may affect the upper urinary tract with development of vesicoureteral reflux. The objective of our study is to evaluate what lower urinary tract dysfunctions are associated with vesicoureteral reflux in adults.

METHODS: We performed a cross-sectional study in a sample of 236 patients (149 males and 87 females) with a mean age of $45.5 \mathrm{yr}$. (typical deviation $19.0 \mathrm{yr}$.) submitted for videourodynamic study for lower urinary tract functional symptoms. History and neurourologic physical examination, filling cystometry associated with videocystography, pressure/flow study associated with voiding videocystography and selective electromyography of the periurethral sphincter were done in all patients. The chisquare test was used for statistical analysis.

RESULTS: The presence of vesicoureteral reflux was observed in 19 patients (8.1\% of the sample). Fifteen cases (79\%) showed reflux during the filling phase, 3 cases $(16 \%)$ during the voiding phase, and in $115 \%)$ in both. The existence of a vesicourethral neurogenic dysfunction was confirmed in 8 cases $(42 \%)$; there was no neurogenic dysfunction in 11 cases (58\%). The vesicoureteral reflux was classified as primary passive (without any urodynamic anomaly) in one case (5\%), passive with diminished compliance in 4 cases (21\%), passive evolutive in 3 cases (16\%), active involuntary in 7 cases $(38 \%)$, active associated with organic obstruction of the lower urinary tract in one case (5\%), active associated with increased contractile potency in one case (5\%), and secondary to abdominal press in 2 cases (10\%). The only urodynamic anomalies associated with a significant increase of the prevalence of vesicoureteral reflux were: a filling pressure at maximum capacity greater than $12.5 \mathrm{H}_{2} \mathrm{O} \mathrm{cm}$ (prevalence of reflux 7 times) and voiding with abdominal press which increased the prevalence of reflux 2.8 times.

CONCLUSIONS: Most patients with functional symptoms of the lower urinary tract and associated vesicoureteral reflux present lower urinary tract dysfunctions that may justify their reflux. Nevertheless, a statistically significant increase of the prevalence of vesicoureteral reflux was only found in patients with filling pressures at maximum capacity greater than $12.5 \mathrm{~cm} \mathrm{H}_{2} \mathrm{O}$ and those voiding with the help of abdominal press.

Keywords: Vesicoureteral reflux. Urodynamics. Risk factors.

\section{INTRODUCCIÓN}

El reflujo vesicoureteral es la inversión del sentido del flujo urinario, que asciende desde la vejiga hasta el tracto urinario superior. La prevalencia de reflujo vesicoureteral en la población infantil con infecciones urinarias de repetición o mielodisplasia es superior al $40 \%$ (1). En adultos, la prevalencia del reflujo disminuye considerablemente, siendo de un $5 \%$ en pacientes sin alteraciones urodinámicas, pero aumentando hasta el $15 \%$ en aquéllos que presentan algún tipo de disfunción del tracto urinario inferior (2).

El reflujo vesicoureteral se asocia a una alteración del gradiente de presiones entre el tracto urinario superior e inferior. Para algunos autores esta alteración del gradiente barométrico se debe a un aumento de la presión intravesical asociado a una alteración urodinámica del tracto urinario inferior (2), (3). Basándose en esta hipótesis, Salinas et al (4), realizan una clasificación del reflujo vesicoureteral según el tipo de alteración urodinámica asociada.

El objetivo de nuestro trabajo es comprobar qué tipo de relación existe entre las alteraciones urodinámicas y el reflujo vesicoureteral en pacientes adultos.

\section{MATERIAL Y MÉTODOS}

Se realizó un estudio transversal ("cross sectional study") en una muestra de 236 pacientes (149 varones y 87 mujeres), de edad media 45,5 años (desviación típica 19,0 años), remitidos para estudio videourodinámico por síntomas funcionales del tracto urinario inferior (T.U.I).

A los pacientes se les realizó una historia clínica y una exploración neurourológica. El estudio videourodinámico consistió en una cistomanometría asociada a videocistografía de llenado, estudio presión/ flujo asociado a videocistografía miccional. En ambos casos se asoció una electromiografía selectiva del esfínter periuretral. Para el estudio se utilizó un equipo MMS $2000 \AA$, asociado a un intensificador de imágenes. El llenado se realizó con una solución de contraste yodado, no iónico, a temperatura ambiente y a un ritmo de llenado de $50 \mathrm{ml} / \mathrm{min}$., mediante un catéter uretral de doble vía $8 \mathrm{~F}$. La presión intravesical se recogió hidráulicamente por otra de las vías del cateter intravesical. La presión abdominal se registro mediante un catéter intrarrectal. La presión del detrusor se obtuvo mediante sustracción electrónica de ambas presiones. Para la electromiografía se utilizaron electrodos superficiales perianales. En caso 
de presunción diagnóstica de disfunción neurógena vesicouretral, se realizó además una electromiografía selectiva mediante electrodo de aguja, procesando la señal en un electromiografo Dantec Cantata ${ }^{\circledR}$.

La acomodación vesical se calculó dividiendo la presión del detrusor (medida en $\mathrm{cm} \mathrm{H}_{2} \mathrm{O}$ ) por la capacidad vesical (volumen vesical al final del llenado, expresado en ml). La hiperactividad vesical se definió como la presencia de contracciones involuntarias del detrusor durante el llenado vesical. El diagnóstico de obstrucción en varones se realizó mediante el diagrama de nomograma de Abrams \& Griffiths en el varón y el nomograma de Blaivas \& Groutz en la mujer. La contractilidad vesical se valoró mediante el cálculo de la potencia a flujo máximo (expresada en miliWatios).

El diagnóstico de reflujo vesicoureteral se realizó durante el estudio videourodinámico. Para su clasificación se utilizó el criterio propuesto por Salinas et al (1992), al que se añadió en el caso del reflujo activo voluntario dos nuevas categorías: activo voluntario con potencia contráctil aumentada, y activo voluntario con obstrucción del TUI. Del reflujo vesicoureteral pasivo primario se eliminaron aquellos pacientes con disfunción neurógena con arreflexia vesical, por considerar que podían corresponder a un reflujo vesicoureteral evolucionado, incluyéndose dentro de una nueva categoría denominada reflujo ureteral pasivo evolutivo.

Para el análisis estadístico se utilizó el test de la chi-cuadrado de comparación de variables categórica y el test de comparación de medias de la † de Student para variables paramétricas. El cálculo del punto de corte de la presión vesical de llenado respecto al diagnóstico de reflujo vesicoureteral se realizó mediante la construcción de una curva de rendimiento diagnóstico (curva ROC).

\section{RESULTADOS}

La acomodación vesical media fue de 81,64 $\mathrm{cm} \mathrm{H}_{2} \mathrm{O} / \mathrm{ml}$ (desviación típica: 112, $19 \mathrm{~cm} \mathrm{H}_{2} \mathrm{O} / \mathrm{ml}$ ). Se observó la presencia de hiperactividad vesical en 106 casos (44,9\%). El valor medio del $n^{\circ}$ de Abrams \& Griffiths (en varones) fue de $28,18 \mathrm{~cm} \mathrm{H}_{2} \mathrm{O}$ (desviación típica: $39,81 \mathrm{~cm} \mathrm{H}_{2} \mathrm{O}$. El nomograma de Blaivas \& Groutz en mujeres indicó ausencia de obstrucción en el $40,2 \%$ de las mujeres, obstrucción leve en $28,7 \%$ de los casos, obstrucción moderada en el $9,2 \%$ de los casos, y obstrucción intensa en el 1,1\% de los casos. El número de pacientes con obstrucción urinaria fue de 41 casos $(17,4 \%)$. La potencia a flujo máximo media de la serie fue de 25, $60 \mathrm{~m}$ Watios.
El número de pacientes diagnosticados de insuficiencia contráctil fue de 70 casos $(29,7 \%)$. El número de paciente con micción utilizando la prensa abdominal fue del 73 casos $(30,9 \%)$.

En 80 casos $(33,9 \%)$, se comprobó la presencia de una disfunción neurógena del TUl. Los tipos de disfunción neurógena se muestran en la Tabla I.

Se observó la presencia de reflujo vesicoureteral en 19 pacientes $(8,1 \%$ de la muestra). En 15 casos $(79 \%)$ el reflujo tuvo lugar durante el llenado, en 3 casos $(16 \%)$ durante la micción, y en 1 caso $(5 \%)$ en ambas fases. El reflujo vesicoureteral se clasificó como pasivo primario (sin alteración urodinámica que lo justifique) en 1 caso (5\%), pasivo por acomodación disminuida en 4 casos $(21 \%)$, pasivo evolutivo en 3 casos (16\%), activo involuntario en 7 casos $(38 \%)$, activo asociado a obstrucción orgánica del TUI en 1 caso $(5 \%)$, activo asociado a potencia contráctil elevada en 1 caso $(5 \%)$, y con prensa abdominal en 2 casos (10\%).

La curva de rendimiento diagnóstico para calcular el punto de corte de la presión vesical de llenado se muestra en la Figura 1. Este punto de corte se estableció en $12,5 \mathrm{~cm} \mathrm{H}_{2} \mathrm{O}$. La relación entre los diferentes datos urodinámicos y la presencia de reflujo vesicoureteral se muestra en la Tabla II.

\section{Curva COR}

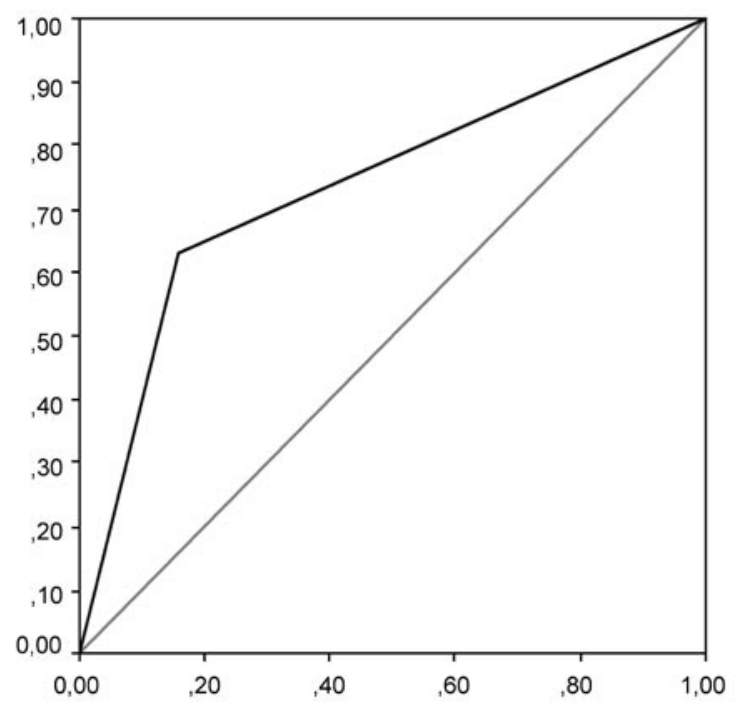

FIGURA 1. Curva de rendimiento diagnóstico de la presión vesical de llenado respecto al reflujo vesicoureteral. 


\section{DISCUSIÓN}

El Principio Fundamental de la Hidrodinámica indica que los fluidos circulan desde el área de mayor presión a la de menor presión. En el caso del reflujo vesicoureteral, hay una inversión del gradiente de presión que hace que la orina refluya desde el tracto urinario inferior al superior. Salinas et al (4), clasificaron las alteraciones urodinámicas del tracto urinario inferior asociadas al reflujo vesicoureteral en cinco tipos: 1). Reflujo vesicoureteral sin alteraciones urodinámicas (primario), y que corresponde al producido por una anomalía de la unión ureterovesical. 2) Reflujo vesicoureteral sin contracción vesical (pasivo) por baja acomodación vesical, 3). Reflujo vesicoureteral con contracción vesical (activo) asociado a hiperactividad vesical (activo involuntario), 4). Reflujo vesicoureteral activo sin hiperactividad vesical (activo voluntario), y 5). Reflujo vesicoureteral asociado a micción con prensa abdominal.

La acomodación vesical es una magnitud relacionada con las propiedades viscoelásticas de la pared vesical. En condiciones normales, el tejido vesical es viscoelástico, permitiendo una progresiva distensión de sus paredes, sin el correspondiente aumento de su tensión (5). Cuando se alteran estas propiedades, debido a la sustitución del músculo liso por colágeno, o a un incremento del tono muscular por lesión simpática (6), la presión vesical aumenta, facilitando de esta manera la inversión del gradiente de presión y el reflujo vesicoureteral. En nuestro estudio, observamos una importante correlación entre la presión vesical de llenado y la existencia del reflujo. El punto de corte a partir del cual el diagnostico de reflujo vesicoureteral es más fiable (máxima sensibilidad y especificidad), se estableció en una presión vesical de llenado de $12,5 \mathrm{~cm} \mathrm{H}_{2} \mathrm{O}$. En nuestro estudio, los pacientes con presiones superiores a la indicada, presentaron una prevalencia de reflujo vesicoureteral 7 veces mayor que los que tenían presiones de llenado inferiores. La baja acomodación vesical, también ha sido reconocida, por otros autores, como uno de los factores de riesgo de padecer reflujo vesicoureteral. Experimentalmente, se ha comprobado que hay una relación lineal entre la presión intravesical y el reflujo vesicoureteral (7). Desde el punto de vista clínico, esta relación es más evidente en pacientes con disfunción neurógena congénita (mielodisplasia) (8), o adquirida (lesión medular traumática) (9).

Nuestro estudio no encontró ninguna relación significativa entre la hiperactividad vesical y el reflujo. La hiperactividad vesical también ha sido sñalada por algunos autores como un factor de riesgo para el reflujo vesicoureteral Koff and Murtagh (10) encontraron que el tratamiento de la hiperactividad vesical en niños disminuía significativamente el reflujo vesicoureteral asociado. También se ha comprobado esta relación en niños con mielodisplasia (8). Sin embargo, en adultos con lesión medular, no ha podido ser demostrada una relación entre la hiperreflexia y el reflujo (11). Nielsen (12) comprueba que existe una tendencia hacia la disminución de la fuerza de asociación entre hiperactividad vesical y reflujo conforme aumenta la edad de los niños. Es posible que la hiperactividad vesical solo sea un factor de riesgo para el reflujo vesicoureteral en la infancia.

TABLA I. DESCRIPCIÓN DE LOS TIPOS DE DISFUNCIONES NEURÓGENAS VESICOURETRALES.

\begin{tabular}{|c|c|c|}
\hline Tipo disfunción neurógena & Número de casos & Porcentaje \\
\hline Neurona motora superior con lesión simpática & 6 & $7 \%$ \\
\hline Neurona Motora superior sin lesión simpática & 31 & $39 \%$ \\
\hline Neurona Motora Inferior con lesión simpática & 3 & $4 \%$ \\
\hline Neurona Motora Inferior sin lesión simpática & 7 & $9 \%$ \\
\hline Hiperreflexia aislada & 3 & $4 \%$ \\
\hline Arreflexia aislada & 27 & $34 \%$ \\
\hline Arreflexia con lesión simpática & 2 & $2 \%$ \\
\hline Arreflexia y lesión pudenda superior & 1 & $1 \%$ \\
\hline Total & 80 & $100 \%$ \\
\hline
\end{tabular}


Nuestro estudio no demostró ninguna asociación entre la potencia contráctil y la obstrucción urinaria con el reflujo vesicoureteral. Capitanucci et al (13) encuentran una relación entre la presión máxima del detrusor durante la micción y el reflujo vesicoureteral en niños. Estos autores consideran que la hipercontractilidad vesical está asociada al reflujo vesicoureteral en niños. Chandra and Maddix (14), también encuentran esta asociación en niños, pero lo atribuyen a la presencia de micción no coordinada. El aumento de presión del detrusor durante la fase miccional también puede ser debida a obstrucción del tracto urinario inferior. Experimentalmente, se ha comprobado que la obstrucción del tracto urinario inferior origina reflujo vesicoureteral en el caso de obstrucción crónica asociada a hiperactividad vesical. Algunos autores refieren la asociación de reflujo vesicoureteral y obstrucción del cuello vesical en mujeres (15). Sin embargo, la mayor asociación de reflujo vesicoureteral y obstrucción urinaria ocurre en pacientes con disinergia de- trusor-esfínter periuretral por disfunción neurógena, (3), (8). En nuestro estudio, los 33 pacientes con disinergia presentaron una prevalencia de reflujo vesicoureteral ligeramente superior $(12,1 \%)$, a los pacientes sin disinergia $(7,4 \%)$, pero sin alcanzar la significación estadística. Es posible que un estudio transversal de prevalencia pueda enmascarar esta asociación.

La asociación entre micción con prensa abdominal y reflujo vesicoureteral resultó significativa en nuestro estudio. Aunque no existen otros estudios que confirmen esta relación en pacientes sin lesión neurógena, Greenstein et al (16) si que refieren que en pacientes con arreflexia vesical la micción con prensa, con presiones superiores a $95 \mathrm{~cm} \mathrm{H}_{2} \mathrm{O}$ es un factor de riesgo para el reflujo. Este dato podría explicar el reflujo vesicoureteral en pacientes con arreflexia (denominado en nuestro estudio reflujo pasivo evolucionado), aunque son precisos estudios prospectivos para confirmar esta relación.

TABLA II. RELACIÓN ENTRE LOS DATOS URODINÁMICOS Y LA PRESENCIA DE REFLUJO.

\begin{tabular}{|c|c|c|}
\hline & Porcentaje de pacientes con reflujo & Nivel de significación \\
\hline Presión de llenado & & $0,000^{*}$ \\
\hline$>12,5 \mathrm{~cm} \mathrm{H}_{2} \mathrm{O}$ & $26,1 \%$ & \\
\hline$<=12,5 \mathrm{~cm} \mathrm{H}_{2} \mathrm{O}$ & $3,7 \%$ & \\
\hline Hiperactividad vesical & & 0,496 \\
\hline $\mathrm{Si}$ & $7,5 \%$ & \\
\hline No & $8,5 \%$ & \\
\hline Obstrucción TUI & & 0,122 \\
\hline $\mathrm{Si}$ & $2,4 \%$ & \\
\hline No & $9,2 \%$ & \\
\hline Potencia contráctil & & 0,130 \\
\hline Normal & $9,6 \%$ & \\
\hline Disminuida & $4,3 \%$ & \\
\hline Micción con prensa & & $0,026^{*}$ \\
\hline $\mathrm{Si}$ & $11,5 \%$ & \\
\hline No & $5,1 \%$ & \\
\hline Disfunción neurógena & & 0,291 \\
\hline $\mathrm{Si}$ & $10 \%$ & \\
\hline No & $7,1 \%$ & \\
\hline
\end{tabular}




\section{BIBLIOGRAFÍA y LECTURAS RECOMENDADAS ( ${ }^{*}$ lectura de interés $y^{* *}$ lectura fundamental)}

*1. SZABO, L.; LOMBAY, B.; BORBAS, E. y cols.: "Videourodynamics in the diagnosis of urinary tract abnormalities in a single center". Pediatr. Nephrol., 19: 326, 2004.

2. CHAPPLE, C.R.; CHRISTMAS, T.J.; TURNERWARWICK, R.T.: "Vesicoureteric reflux in the adult male". Br. J. Urol., 65: 144, 1990.

3. SERUCA, H.: "Vesicoureteral reflux and voiding dysfunction: a prospective study". J. Urol., 142: 494, 1989.

**4. SALINAS CASADO, J.; PRIETO CHAPARRO, L.; PAEZ BORDA, A. y cols.: "Nueva clasificación del reflujo vesicoureteral". Arch. Esp. Urol., 45: 449, 1992

5. SALINAS, J.; VÍRSEDA, M.; FUENTE, M.P. y cols.: "A study on the viscoelastic properties of the urinary bladder in dogs". Urol. Inter., 49: 185, 1992.

*6. ADOT ZURBANO, J.M.; SALINAS CASADO, J.; VALER ALGARABEL, J. y cols.: "Participación funcional del componente simpático D10-L2 en la dinámica del tracto urinario inferior". Arch. Esp. Urol., 56: 385, 2003.

7. ANGELL, S.K.; PRUTHI, R.S.; SHORTLIFFE, L.D.: "The urodynamic relationship of renal pelvic and bladder pressures, and urinary flow rate in rats with congenital vesicoureteral reflux". J. Urol., 160: 150, 1998.

8. KOBAYASHI, S.; SHINNO, Y.; KAKIZAKI, H. y cols.: "Relevance of detrusor hyperreflexia, vesical compliance and urethral pressure to the occurrence of vesicoureteral reflux in myelodysplastic patients". J. Urol., 147: 413, 1992.

9. GERRIDZEN, R.G.; THIJSSEN, A.M.; DEHOUX, E.: "Risk factors for upper tract deterioration in chronic spinal cord injury patients". J. Urol., 147: 416, 1992.

10. KOFF, S.A.; MURTAGH, D.S.: "The uninhibited bladder in children: effect of treatment on recurrence of urinary infection and on vesicoureteral reflux resolution". J. Urol., 130: 1138, 1983.

11. LINSENMEYER, T.A.; BAGARIA, S.P.; GENDRON, B.: "The impact of urodynamic parameters on the upper tracts of spinal cord injured men who void reflexly". J. Spinal Cord. Med., 21: 15, 1998.

12. NIELSEN, J.B.: "Lower urinary tract function in vesicoureteral reflux". Scand. J. Urol. Nephrol. Suppl., 125: 15, 1989.

13. CAPITANUCCI, M.L.; SILVERI, M.; MOSIELLO, G. y cols.: "Prevalence of hypercontractility in male and female infants with vesico-ureteral reflux". Eur. J. Pediatr. Surg., 10: 172, 2000.

14. CHANDRA, M.; MADDIX, H.: "Urodynamic dysfunction in infants with vesicoureteral reflux". J. Pediatr., 136: 754, 2000.

15. PAVLICA, P.; VIGLIETTA, G.; RAMINI, R. y cols.: "Sindrome Ostrutivo dal collo vescicale da la donna". Radiol. Med. (Torino), 77: 496, 1989.

16. GREENSTEIN, A.; RUCKER, K.S.; KATZ, P.G.: "Voiding by increased abdominal pressure in male spinal cord injury patients-long term follow-up". Paraplegia, 30: 253, 1992. 